\title{
SCIENTIFIC REPORTS

\section{Genome-wide analyses disclose the distinctive HLA architecture and the pharmacogenetic landscape of the Somali population}

\begin{abstract}
Abshir A. Ali' ${ }^{1}$, Mikko Aalto ${ }^{2}$, Jon Jonasson ${ }^{3}$ \& Abdimajid Osman ${ }^{4 *}$
African populations are underrepresented in medical genomics studies. For the Somali population, there is virtually no information on genomic markers with significance to precision medicine. Here, we analyzed nearly 900,000 genomic markers in samples collected from 95 unrelated individuals in the North Eastern Somalia. ADMIXTURE program for estimation of individual ancestries revealed a homogenous Somali population. Principal component analysis with PLINK software showed approximately $60 \%$ East African and $40 \%$ West Eurasian genes in the Somali population, with a close relation to the Cushitic and Semitic speaking Ethiopian populations. We report the unique features of human leukocyte antigens (HLA) in the Somali population, which seem to differentiate from all other neighboring regions compared. Current study identified high prevalence of the diabetes type 1 (T1D) predisposing HLA DR-DQ haplotypes in Somalia. This finding may explain the increased T1D risk observed among Somali children. In addition, ethnic Somalis were found to host the highest frequencies observed thus far for several pharmacogenetic variants, including UGT1A4*2. In conclusion, we report that the Somali population displays genetic traits of significance to health and disease. The Somali dataset is publicly available and will add more information to the few genomic datasets available for African populations.
\end{abstract}

Africa harbors the largest human genetic variation in the world ${ }^{1-5}$ and many human gene variants are found only in Africa ${ }^{6}$ including those associated with drug response. Yet, many ethnic populations in Sub-Saharan Africa are not represented in medical genomics studies found in the literature, mainly because of the generally lower level of medical research conducted in Sub-Saharan Africa compared with developed countries due to insufficient research infrastructures or resources. As a result, most of the human genetic variation present in Africa is yet unexplored. Some efforts have been made in recent years to better understand African genetic variation. These include studies that examined genetic markers for diabetes ${ }^{7,8}$ and those investigating the genetic selection in Africa as a result of disease exposure or environmental adaptation ${ }^{4,9}$. However, the need for more genomic data from African populations still persists, notably for underrepresented ethnolinguistic groups, including the Somali population, where there is still lack of information on genetic markers for drug responses, immunity and diseases.

Somalia is located in the Horn of Africa (Somalia, Djibouti, Ethiopia, Eritrea,), a historic site for human migrations into either direction of Africa or Eurasia ${ }^{10-13}$. Rock art paintings in multiple sites of Northern Somalia show Neolithic human activities in this part of East Africa ${ }^{14,15}$. Later Himyarite and Sabaean inscriptions found in the same Somali region suggest existence of socio-cultural mingling with the ancient Axumite-South Arabian sphere ${ }^{15}$. Due to the geographic location of Somalia, the Somalis have both African and non-African ancestries $^{12,13,16,17}$. Most of the genetic studies involving ethnic Somalis in recent years have mainly focused either on forensic genetic markers used for legal issues ${ }^{18-21}$, or on anthropological genetics for assessment of ancestry and ancient demographic history ${ }^{12,16}$, using DNA samples collected from the Somali diaspora outside Africa. While such studies have provided interesting information on the genetic history of Somalia, there is scarce information on genomic data of importance to health and disease for the Somali population, apart from some

${ }^{1}$ Faculty of Medicine, East Africa University, Bosaso, Puntland, Somalia. ${ }^{2}$ Bosaso general hospital, Bosaso, Puntland, Somalia. ${ }^{3}$ Department of Clinical Genetics, and Department of Biomedical and Clinical Sciences, Linköping University, Linköping, Sweden. ${ }^{4}$ Department of Clinical Chemistry, and Department of Biomedical and Clinical Sciences, Linköping University, Linköping, Sweden. *email: majid.osman@liu.se 
A

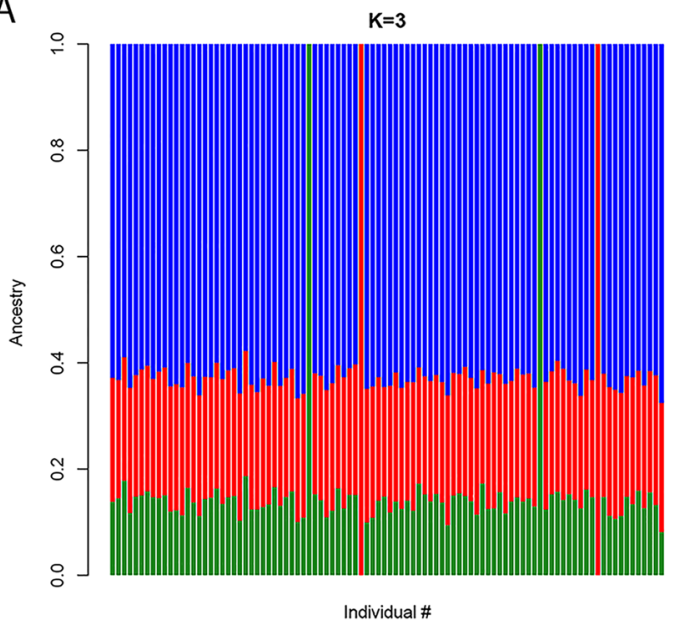

B

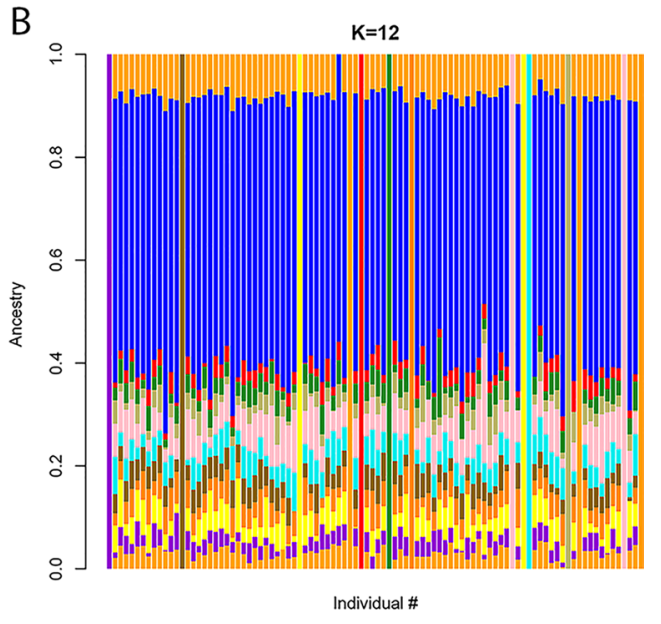

Figure 1. Maximum likelihood estimation of the ethnic descent of the 95 Somali individuals in this study. Data used comprised 849,267 markers in the Axiom PMRA multilocus SNP genotyping. PLINK 1.9 with a setting of $\mathrm{MAF} \geq 0.4$ was used along with analysis by the ADMIXTURE 1.3.0 program with $\mathrm{K}=3$ and $\mathrm{K}=12$, respectively (http://software.genetics.ucla.edu/admixture/). (A) $\mathrm{K}=3$ proportions represent East-African genes (blue), genes from West Asia (red), and North African genes (green), respectively. (B) K=12 splits the supposedly non-African contributions into several minor bands (mixed colors), although the similarity of individuals is well preserved.

single-gene-disorder case reports involving Somali patients living in Western countries. As an example, there are no datasets available concerning pharmacogenetics of drug metabolizing enzymes and transporters (DMET), HLA alleles, or disease markers for the Somali population. This lack of genomic information can be partly, but not wholly, explained by the political situation in the country, which has discouraged local and international researchers to conduct medical research during the turbulent years of Somalia. The few genetic case reports available in the literature, nevertheless, suggest that Somalia harbors some unique human genetic traits with implications to health and disease. These include the exceptionally high prevalence (93\%) of the human leukocyte antigen (HLA) DRB1*03:01 allele found in Somali children with type 1 diabetes (T1D) in Minnesota ${ }^{22}$, the high frequency (75\%) of the T1D predisposing HLA DR3-DQ2 haplotypes identified in Somali children with T1D in Finland ${ }^{23}$, the founder effect in the Horn of Africa of the insulin receptor mutation p.Ile119Met identified in unrelated Somali families living in the United Kingdom ${ }^{24}$, and the high incidence rate of mutations in adenosine deaminase (ADA) causing ADA-deficiency observed in Somali immigrants living in Denmark ${ }^{25}$. In the present study, we have tried to address the aforementioned needs for a Somali genomic data and for the first time created a genome-wide, publicly accessible, dataset containing genomic markers with significance to precision medicine for this ethnic population.

\section{Results}

Array analysis summary. A total number of 95 samples and 1 Quality Control sample (included in the PMRA reagent) were analyzed on the GeneTitan MC Instrument. All samples passed the Dish QC (DQC) test. There were 888,799 markers analyzed in the study. Average genotype quality control call rate (QC CR) was 99.6\%, which generated 849,267 high-quality markers that were included in the downstream analyses. Gender call counts (male $=57$; female $=38$; unknown $=0$ ) matched perfectly with the documented gender identity for study participants. Results for QC analyses are available in Supplementary Fig. 1-4 (Supplemental Data file).

Genotype data verification. To validate the genotype data, we examined markers known to associate with ethnic and geographic variations that were included in the PMRA analysis. First, we estimated the distribution of ABO and Rh blood groups in our Somali population using a set of 8 SNPs: rs8176719, rs8176746, rs505922, rs8176749, rs7853989, rs8176740, rs56392308 (ABO blood groups), and rs590787 (Rh blood type). The most common ABO blood group in Somalia was found to be O blood (60\%), followed by A blood group (22\%, of which $14 \%$ of the total A belonged to A2 type), B blood group (14\%), and AB blood group (4\%). The proportion of $\mathrm{Rh}+$ and $\mathrm{Rh}$ - were $88 \%$ and $12 \%$, respectively. These results were consistent with the previously reported Somali phenotype blood group data ${ }^{26}$ as well as with our previous $\mathrm{O}$ blood group genotype assessments for ethnic Somalis ${ }^{27}$. We also examined the distribution of Apolipoprotein E (ApoE) gene variants in the Somali population using the SNPs rs429358 and rs7412. ApoE is a genetic and anthropological marker that shows geographical and ethnic variations ${ }^{28}$. Our analysis showed that the most common ApoE gene variant in Somalia is ApoE- 83 (79\%), followed by ApoE- $\varepsilon 4$ (16\%), and ApoE- $\varepsilon 2$ (6\%). Homozygosity for the $\varepsilon 4$ variant (ApoE- $\varepsilon 4 / \varepsilon 4)$ reported to show the strongest association with Alzheimer's disease in western countries ${ }^{29}$ was found at $2 \%$ in Somalia. These ApoE gene frequencies are close to those previously identified in the neighboring Ethiopia ${ }^{28,30,31}$.

Principal component analysis. The ADMIXTURE results (Fig. 1) indicate that the subjects of this study constitute a homogeneous and representative sample of Somalis having approximately $60 \%$ East-African and $40 \%$ 


\section{Principal Component Analysis}

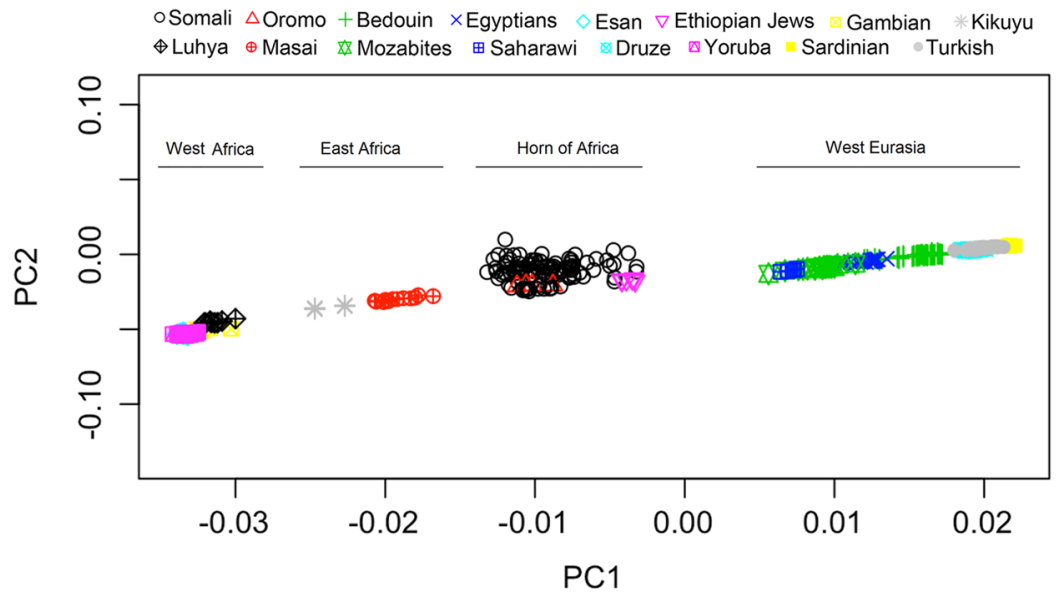

Figure 2. Principal Component Analysis (PCA) of the Axiom PMRA array data from 95 Somali study subjects. Somali data were projected onto African-West Eurasian PC1 and PC2 data from PGG population. The Luhya population in Kenya clustered with West Africans (far left on the diagram) rather than with East Africans.

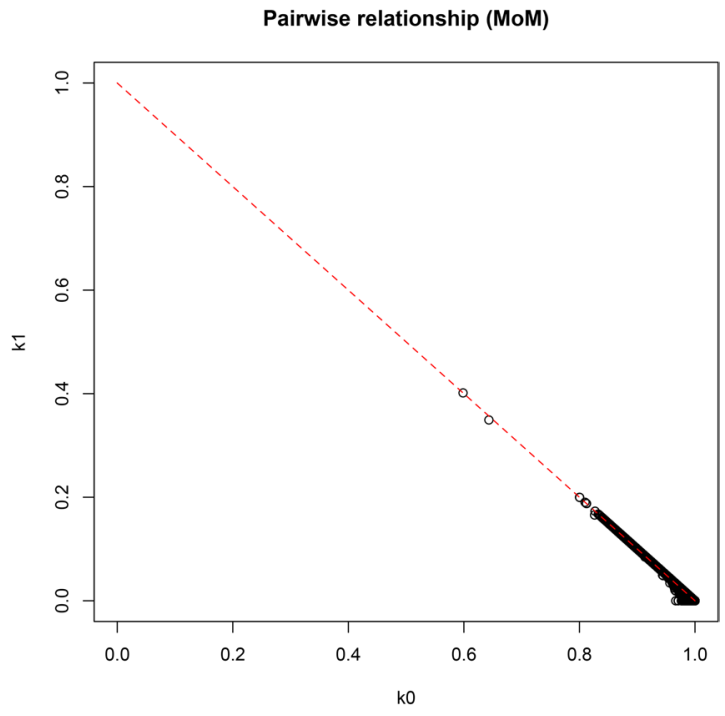

Figure 3. Identity by descent (IBD) analysis performed on the Somali study participants $(n=95)$. The plot shows observed identity by descent for each individual. Each of the 2 outliers shown in the graph was supposedly a first cousin to another individual in the cohort.

West Eurasian gene components. The K3 factor in Fig. 1A corresponds to the ADMIXTURE K10 in Fig. 1A of Hodgson et al ${ }^{16}$ and shows approximately 60\% East African and 40\% West Eurasian (25\% West Asian and 15\% North African) ancestry in the Somali population. The similarity of individuals is apparent, which presumably reflects very ancient admixture events and a unification process through endogamy. In addition, the non-African components in the Somali population seem to show relatively little inter-individual variations (K12 in Fig. 1B), also consistent with the finding of Hodgson et al. ${ }^{16}$. As expected, PCA (Fig. 2) shows a close relation of the study population with the two Ethiopian ethnic groups of Oromo (Cushitic speaking) and Ethiopian Jews (Semitic speaking). The PCA in Fig. 2 shows nearly a straight line relationship between PC1 and PC2 when African and West Eurasian populations are included. To identify the degree of relatedness between each pair of study samples, identity by descent (IBD) analysis was performed. Apart from two outliers who supposedly represented a pair of two pairs of double first cousins, the study cohort did not represent closely related individuals (Fig. 3). No first degree relationship was observed. The mean kinship coefficient was 0.011 .

Drug metabolizing enzymes and transporters (DMET) variants. More than 1200 pharmacogenetic variants were analyzed in the Somali population, of which we identified 631 clinically relevant DMET common variants listed in the PharmGKB database (https://www.pharmgkb.org/) (Supplementary Table 1 file). Comparing with the data found in the Genome Aggregation Database (https://gnomad.broadinstitute.org/) and the 1000 


\begin{tabular}{|c|c|c|c|c|c|c|c|c|c|}
\hline SNP ID & $\begin{array}{l}\text { Associated } \\
\text { gene }\end{array}$ & $\mathbf{R A}^{\mathbf{a}}$ & $\mathbf{A A}^{\mathbf{b}}$ & $\begin{array}{l}\mathrm{AA}^{\mathrm{b}} \\
\text { frequency } \\
\text { Somalia }\end{array}$ & $\begin{array}{l}\mathrm{AA}^{\mathrm{b}} \text { frequency } \\
\text { Sub-Saharan } \\
\text { Africa }^{\mathrm{c}}\end{array}$ & $\begin{array}{l}\mathrm{AA}^{\mathrm{b}} \\
\text { frequency } \\
\text { GnomAD }^{\mathbf{d}}\end{array}$ & $\begin{array}{l}\text { Type } \\
\text { of drug } \\
\text { effect }\end{array}$ & Drug & phenotypes \\
\hline rs61742245 & VKORC1 & $\mathrm{C}$ & A & 0.084 & 0.001 & 0.002 & Dosage & warfarin & Thromboembolism \\
\hline rs6755571 & UGT1A4 & $\mathrm{C}$ & A & 0.137 & 0.007 & 0.035 & Other & ABT-751 & Neoplasms \\
\hline rs9901675 & CD68 & G & A & 0.432 & 0.076 & 0.054 & Efficacy & methylphenidate & $\begin{array}{l}\text { Attention Deficit Disorder } \\
\text { with Hyperactivity }\end{array}$ \\
\hline rs9901673 & CD68 & C & A & 0.463 & 0.166 & 0.168 & Efficacy & Methylphenidate & $\begin{array}{l}\text { Attention Deficit Disorder } \\
\text { with Hyperactivity }\end{array}$ \\
\hline rs16950650 & $\mathrm{ABCC} 4$ & $\mathrm{C}$ & $\mathrm{T}$ & 0.300 & 0.121 & 0.047 & Efficacy & cisplatin, irinotecan & Carcinoma, Small Cell \\
\hline rs5918 & ITGB3 & $\mathrm{T}$ & C & 0.221 & 0.092 & 0.121 & Efficacy & aspirin & $\begin{array}{l}\text { Acute coronary syndrome, } \\
\text { Coronary Artery Disease }\end{array}$ \\
\hline rs757978 & FARP2 & C & $\mathrm{T}$ & 0.205 & 0.108 & 0.101 & Efficacy & methylphenidate & $\begin{array}{l}\text { Attention Deficit Disorder } \\
\text { with Hyperactivity }\end{array}$ \\
\hline rs 17238540 & HMGCR & $\mathrm{T}$ & G & 0.202 & 0.108 & 0.037 & Efficacy & pravastatin & Hypercholesterolemia \\
\hline rs983332 & - & G & $\mathrm{T}$ & 0.495 & 0.318 & 0.206 & Efficacy & $\begin{array}{l}\text { tumor necrosis factor alpha } \\
\text { (TNF-alpha) inhibitors }\end{array}$ & Arthritis. Rheumatoid \\
\hline
\end{tabular}

Table 1. Nine SNPs listed in the PharmGKB database (https://www.pharmgkb.org/) with the highest frequencies in Somalia compared with other world populations. Information on variant-drug-phenotype association was available on the PharmGKB. ${ }^{a}$ reference allele; balternative allele; ${ }^{c} 1000$ Genome Project; ${ }^{\mathrm{d}}$ Genome Aggregation Database.

\section{VKORC1 haplotypes}

A)

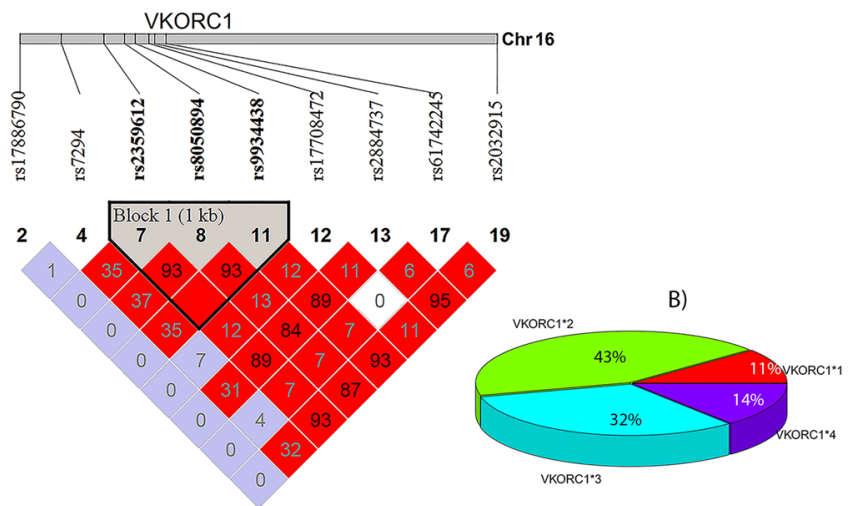

Figure 4. Haplotype structure of the $V K O R C 1$ gene in the Somali population. (A) A region of 5805 base pairs in the VKORC1 gene is shown with tag SNPs for different VKORC1 haplotypes. The SNPs rs2359612, rs8050894, and rs 9934438 previously reported to be part of $V K O R C^{*} 2$ haplotype and found to be predictive of warfarin dose requirement in both European and Asian-descent individuals are in linkage disequilibrium in the Somali population. SNP rs61742245 represents VKORC1 Asp36Tyr associated with warfarin resistance. 16 of 95 Somali individuals were heterozygous to this variant. (B) Proportion of VKORC1 haplotypes in the Somali population.

genomes project (https://www.internationalgenome.org/), a set of 9 pharmacogenetically relevant SNPs was found with unusually high prevalence in Somalia when compared with all other world populations (Table 1). We found the highest frequency of UDP-glucuronosyltransferase 1A4 *2 (UGT1A4*2; rs6755571; p.Pro24Thr) reported to date in the Somali cohort. UGT1A4 catalyzes the conjugation of a wide range of xenobiotic and endogenic substrates ${ }^{32}$ and UGT1A $4 * 2$ was found to correlate with altered glucuronidation of various drugs ${ }^{33}$. Although homozygosity to UGT1A $4 * 2$ is rare in other populations, 3 individuals (3\%) were homozygous to this variant in our Somali cohort, which along with 20 heterozygotes suggested that $24 \%$ of the Somalis carry UGT1A $4 * 2$ with an allele frequency of $14 \%$ (Table 1). This study also identified VKORC1 Asp36Tyr (rs61742245) previously reported to be associated with warfarin resistance in Ethiopians ${ }^{34}$. In our Somali population, 16 individuals (17\%) were heterozygous to VKORC1 Asp36Tyr corresponding to a minor allele frequency (MAF) of $8 \%$ (Table 1).

VKORC1 has been extensively studied in recent years since its discovery ${ }^{35,36}$ in 2004, and we used Haploview software to examine its haplotype structure in the Somalis using tag SNPs discriminating VKORC1*2, VKORC1*3, and $V K O R C 1 * 4$ haplotypes, as we previously described ${ }^{37}$. Our analysis suggested that VKORC1 haplotype frequencies among ethnic Somalis were close to those observed in West Eurasians ${ }^{38,39}$. Three SNPs, rs 2359612 , rs8050894 and rs9934438, previously reported to be part of the VKORC1*2 haplotype and being associated with lower warfarin dose requirement ${ }^{38}$, were found to be in linkage disequilibrium in the Somali population (Fig. 4A). The most common VKORC1 haplotype in Somalia was VKORC1*2 (43\%), followed by VKORC1*3 


\begin{tabular}{|c|c|c|}
\hline HLA & \begin{tabular}{|l|} 
Class \\
\end{tabular} & Allele frequency \\
\hline DPA $1 * 01: 03$ & II & 0.621 \\
\hline DQA1*05:01 & II & 0.371 \\
\hline DQB1*02:01 & II & 0.363 \\
\hline DRB3*02:02 & II & 0.342 \\
\hline DPB1*02:01 & II & 0.330 \\
\hline DRB1*03:01 & II & 0.315 \\
\hline $\mathrm{B} * 07: 02$ & I & 0.260 \\
\hline $\mathrm{A}^{*} 02: 01$ & I & 0.234 \\
\hline DQA1*01:02 & II & 0.229 \\
\hline C*07:02 & I & 0.227 \\
\hline DRB1*13:02 & II & 0.208 \\
\hline DRB3*03:01 & II & 0.207 \\
\hline$A * 01: 03$ & I & 0.199 \\
\hline DPB1*04:01 & II & 0.198 \\
\hline $\mathrm{DPA}{ }^{*} 02: 01$ & II & 0.190 \\
\hline DQA1*01:01 & II & 0.188 \\
\hline DQB1*05:01 & II & 0.181 \\
\hline DQB1*06:04 & II & 0.175 \\
\hline DPB1*03:01 & II & 0.160 \\
\hline $\mathrm{C}^{*} 07: 01$ & I & 0.148 \\
\hline
\end{tabular}

Table 2. The top 20 most frequent HLA alleles (4-digits resolution) in Somalia.

$(32 \%)$ and $V K O R C 1 * 4(14 \%)$. The VKORC1*1 haplotype previously reported to be the ancestral haplotype ${ }^{38}$ had a frequency of $11 \%$ in Somalia (Fig. 4B). All SNPs were in concordance with Hardy-Weinberg equilibrium.

Human leukocyte antigen (HLA) alleles. We evaluated >9,000 HLA markers with the Axiom HLA Analysis software to infer HLA alleles from 11 major MHC Class I and Class II HLA loci. After quality filtering, 94 HLA alleles in a 4-digit resolution were compiled for the Somali population (Supplementary Table 2, in the Supplemental Data file), of which 5 were listed in the PharmGKB database: HLA-A*02:01, HLA-B*58:01, HLA-C*18:01, HLA-DQA $1 * 02: 01$ and HLA-DRB1*03:01. In Table 2, the 20 most frequent HLA alleles in Somalia are shown. To compare the Somali HLA data with those of other populations, we applied hierarchical clustering and PCA using populations available on the Allele Frequency Net Database (AFND; http://www.allelefrequencies.net/). Of the 94 HLA alleles identified in Somalia, 61 were available on the AFND for all populations compared, and these 61 HLA were used for the analysis (Supplementary Table 3, in the Supplemental Data file). As can been in Fig. 5A, the Somali samples clustered separately from other populations. Some HLA alleles were particularly more frequent in Somalia than in any other geographic location compared, including the T1D predisposing DRB1*03:01, previously reported to be highly frequent in Somali children with $\mathrm{T}_{1} \mathrm{D}^{22}$ and also found to be associated with autoimmune hepatitis type- $1^{40}$. We also performed PCA to examine the relative genetic distance in HLA type between different populations. Although we found Ethiopia to be the closest genetic neighbor to Somalia with regard to HLA, the Somali population deviated from other populations in the PCA (Fig. 5B). The Somalis had also the largest contribution to PCA dimensions of all populations, twice more than the next population (Fig. 5C).

Finally, we investigated the correlation in gene frequencies for HLA and DMET between Somalia and Ethiopia, using the abovementioned 61 HLA alleles as well as a group of 15 DMET alleles previously studied in Ethiopia ${ }^{41-45}$. We found that DMET frequencies correlated better than HLA frequencies between the two populations (Fig. 6).

\section{Discussion}

Numerous genetic variants associated with drug response and disease susceptibility have been identified in the past in different populations. Unfortunately, African populations are underrepresented in these studies despite most of the human genetic variation being present in Africa ${ }^{1-5,9}$. In this context, we have created a genome-wide and publicly accessible Somali dataset using Axiom PMRA SNP array. The Somali people speak the Somali language, an Eastern branch of the Cushitic language family that is a division of the great Afro-Asiatic phylum. Unsurprisingly, our Somali samples clustered with other Horn of African populations (Oromo and Ethiopian Jews), mirroring the geographic location of Somalia. Ethnic Somalis, approximately 30 million worldwide, are unified by a shared culture, language and religion. Our data reveal a remarkably homogeneous Somali population with roots from ancient mixtures of populations from Africa and the Middle East. We observed that a vast majority of our study individuals carried similar proportions of genes from those ancient populations, which possibly can be explained by the fact that ethnic Somalis have a strong genetic unification by endogamy, due to the custom of marrying only within the limits of their ethnic group, in addition to consanguineous practices ${ }^{46}$. It is worth to mention that most of our samples were collected in the North Eastern region (Puntland) and that results in this study may reflect the genetic makeup of the population in this part of Somalia. However, recent evidences based 
A

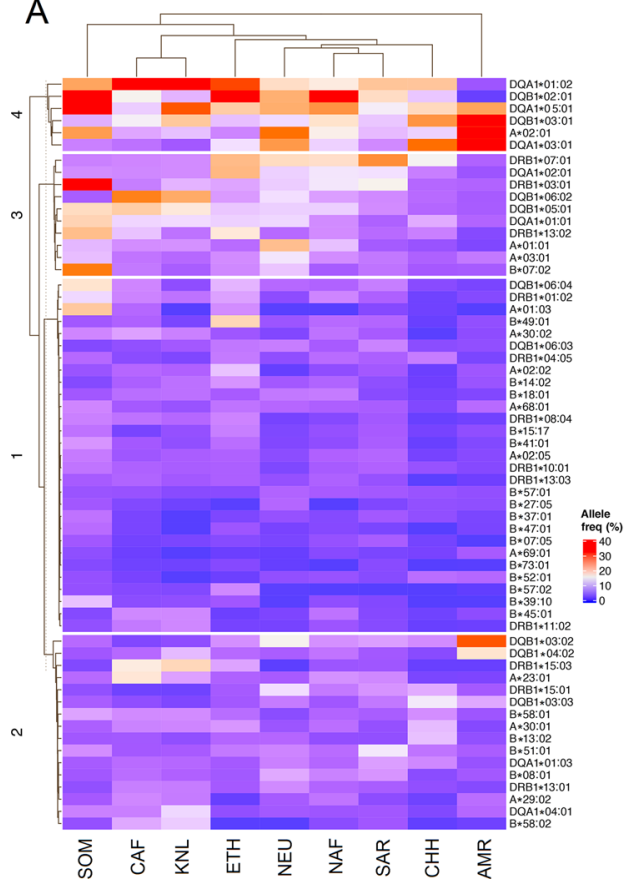

B
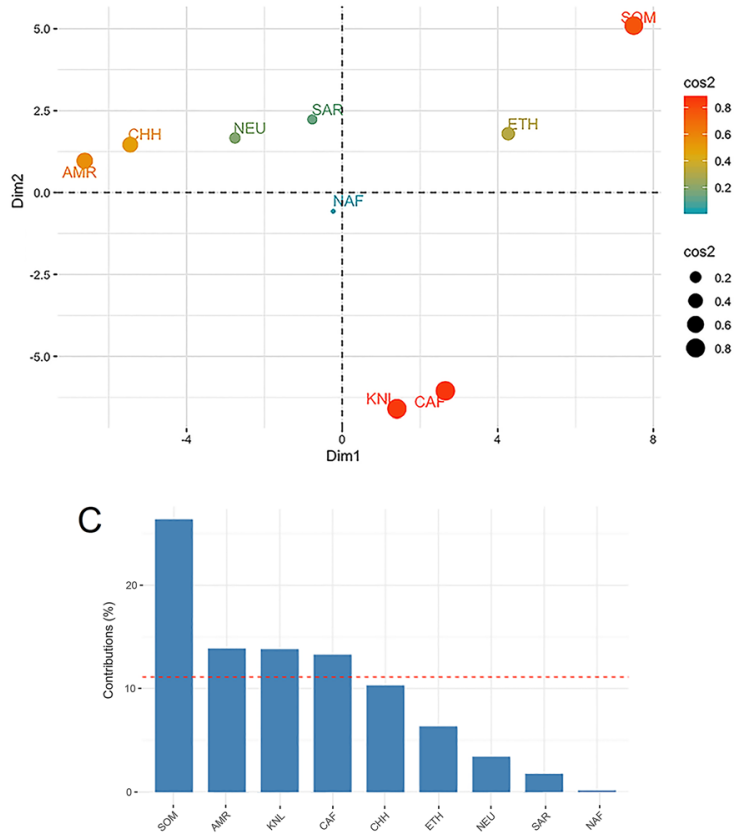

Figure 5. HLA Allele frequencies in different world populations, including Somalis. (A) Hierarchical population clustering of $61 \mathrm{HLA}$ alleles in different world populations. Somalia clustered separately from neighboring countries/populations. (B) Principal component analysis (PCA) using the same set of 61 HLA alleles. Color and size of cos2 signify the quality of representation; the larger a circle is the greater is its contribution to the variables. It is worth to note the considerable variation between African populations compared to non-African populations who seem to be closer to one another. (C) Population contribution to the first two principal components. The Somali population has the largest contribution to the first two dimensions. The Ethiopian population in the analysis did not include Ethiopian Somalis, but rather comprised Oromo, Amhara and Ethiopian Jews. Populations compared were Somalia (SOM), Central Africa (CAF), Kenyan Luo tribe (KNL), Ethiopia (ETH), North Africa (NAF), South Arabia (SAR), North Europe (NEU), Han Chinese $(\mathrm{CHH})$, and Native Americans (AMR).

on Y-STR haplotypes studied on Somalis from diverse geographic locations and clans suggest that ethnic Somalis are largely homogenous ${ }^{17}$, supporting the representativeness of our samples for the larger Somali population. In addition, frequencies of $\mathrm{ABO}$ and Rh blood groups estimated in this study are consistent with those found in an earlier study performed in 1987 by the Somali Red Crescent Society and the Finnish Red Cross Blood Transfusion Service who analyzed 1,026 blood samples of Somalis from the entire country ${ }^{26}$. Although $\mathrm{ABO} / \mathrm{Rh}$ systems tend to show similar distributions for all neighboring populations, our genotypic blood group estimation is remarkably very close to the previously reported Somali phenotypic blood group data, again supporting a representative Somali population in our cohort.

We identified a number of pharmacogenetic gene variants with unusually high prevalence in the Somali population compared with all other populations, although studies involving Somali patients will be required to evaluate the significance of these as well as other variants for drug response in this population. Current study, nevertheless, confirms the Horn of African origin of VKORC1 Asp36Tyr (rs61742245), a variant identified to be associated with warfarin resistance, initially among Ethiopians ${ }^{34}$ and later in other populations in the Middle East, with an allele frequency peaking in Ethiopia ${ }^{47}$. The $8 \%$ MAF for this variant that we found in our Somali population is the second highest frequency of VKORC1 Asp36Tyr reported so far in the world, suggesting that this variant presumably arose in the Horn of Africa and spread into other neighboring regions. This study also found that VKORC1 haplotypes in Somalia are more similar to those found in West Eurasians rather than in other Sub-Saharan Africans, probably reflecting the considerable West Eurasian gene components found in the Somali population. The high frequency of VKORC1 Asp36Tyr and the type of VKORC1 haplotypes found in Somalia have consequences for precision medicine. Although novel oral anticoagulants, or non-vitamin K antagonist oral anticoagulants, (NOACs) are increasingly replacing warfarin for treatment and prophylaxis of venous thromboembolism $(\mathrm{VTE})^{48}$, warfarin is still in use and may likely remain in use for years to come. Thus, Somali VTE patients undergoing warfarin treatment could benefit from genotyping for VKORC1 Asp36Tyr variant as well as for VKORC1 haplotypes to establish a proper warfarin dosing regimen for avoiding unwanted fluctuations in PT/ INR during the initiation period of warfarin.

We found only 15 DMET gene variants genotyped in Ethiopia in the literature, whose frequencies correlated very well with those we identified in Somalia. In contrast, when HLA alleles were compared between the two populations, the correlation was considerably poorer (Fig. 6), suggesting that frequencies of DMET alleles have remained relatively unchanged in the Horn of Africa since the separation of Cushitic and Semitic speaking 
HLA alleles

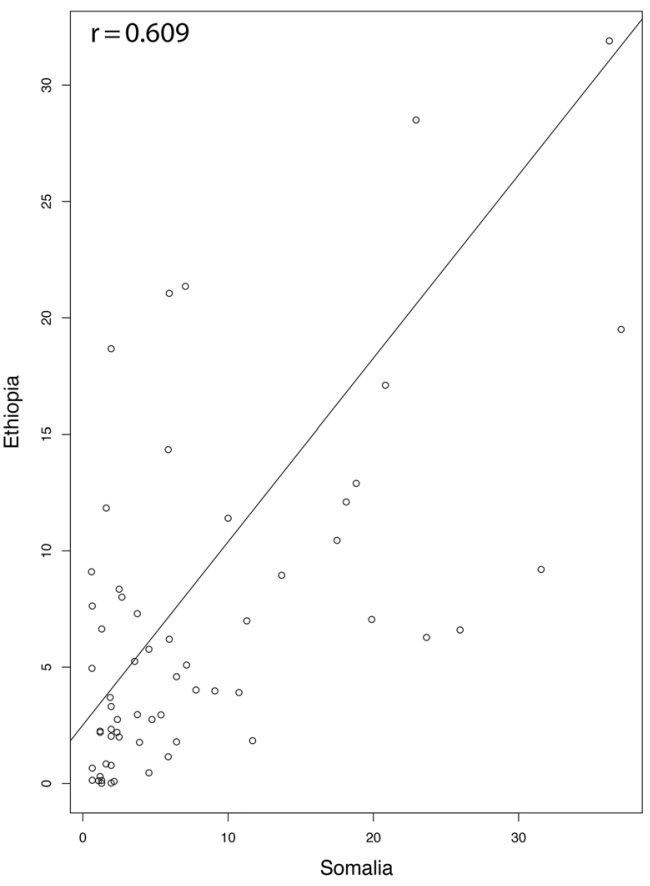

DMET alleles

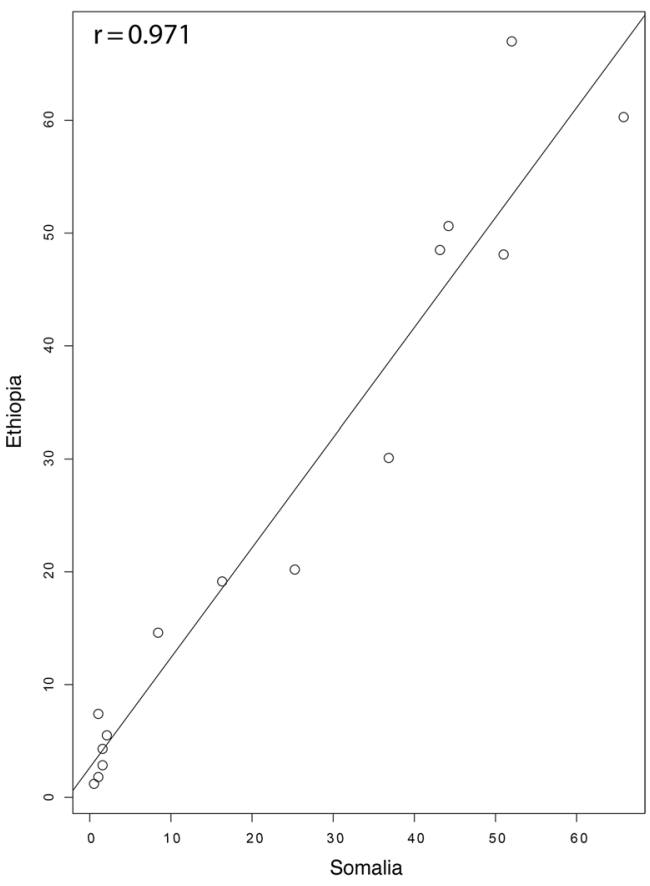

Figure 6. Correlations of HLA $(n=61)$ and DMET $(n=15)$ alleles between Somali and Ethiopian populations. Horizontal and vertical axes represent allele frequencies in each population.

groups, whereas the more extensively polymorphic HLA genes ${ }^{49}$ presumably have been more affected by historical changes in environment and disease burden in the Horn of Africa. Exceptions include DRB1*13:02 and $\mathrm{DRB} 1 * 01: 02$, which show Horn of African signatures with frequencies peaking in Somalia and Ethiopia. It is, however, likely that local adaptations as a result of exposure to differing infectious diseases have resulted in a differentiated HLA architecture in Somalia. There are considerable environmental and geological differences between Somalia and Ethiopia. Whereas Somalia and the Eastern Somali region of Ethiopia are largely arid or semi-arid, there have been historically more favorable conditions for vegetation and farming in larger parts of Ethiopia. Recent studies carried on African populations observed differentiations in genes involved in immunity as a result of altered environment and exposure to diseases ${ }^{4,9}$. As an example, Gurdasani et al. previously reported that HLA-DRB1 is differentiated in some African populations as a result of historical exposure to Lassa fever'. Overall, HLA alleles in Somalia appear to diverge from those in proximal geographic locations as can be seen in the hierarchical heatmap plot (Fig. 5A). Our PCA plot (Fig. 5C) also reveals that the Somali samples contributed the greatest variance to the first two principal components, suggesting that the Somali population has a unique HLA profile.

Remarkably, several HLA alleles with high prevalence in Somalia have been found to be associated with T1D in other studies. In particular, the genotypic combination DRB1*03:01 - DQA1*05:01 - DQB1*02:01 (DR-DQ haplotype) has been found to significantly increase T1D risk ${ }^{50}$. Onengut-Gumuscu et al. ${ }^{8}$ recently reported several ancestry-specific and disease-associated HLA variants. In their study, the MHC class II haplotype DRB ${ }^{*} 0$ 3:01-DQA ${ }^{*}$ 05:01-DQB1*02:01 was a marker for T1D in Caucasians (adjusted odds ratio [OR] $=3.91 ; P=2.6$ $\times 10^{-78}$ ). In our study, the Somali population appears to host the highest frequency in the world for this HLA haplotype. In contrast, African American-specific HLA haplotypes identified by Onengut-Gumuscu et al. ${ }^{8}$ are not prevalent in Somalia. This may be explained by the fact that most African Americans have their African ancestry in West/Central Africa, in populations speaking Niger-Congo languages who are not ethnolinguistically related to the Cushitic-Semitic speaking ethnic groups in the Horn of Africa. Another SNP, rs9273363 (HLA-DQB1-AS1), in the $M H C$ gene with strong association with $\mathrm{T}_{10}^{8}\left(\mathrm{OR}=5.48 ; P=4.61 \times 10^{-138}\right)$ was also found to have the highest allele frequency (AF) in the world in Somalia, with an AF of 0.35 in our cohort. Corresponding AF for SNP rs9273363 in other populations is 0.091 in other Sub-Saharan Africans, 0.331 in Native Americans, 0.319 in East Asians, 0.262 in Europeans, and 0.257 in South Asians, according to the 1000 genomes reference panel (https://www.internationalgenome.org). There is no genomic data for Horn of African populations in the 1000 genomes project. This highlights the considerable genetic diversity existing among populations of African ancestry and the need to include more African ethnic groups in DNA sequencing studies, as we previously stressed ${ }^{2}$. At least 140 million Cushitic and Semitic speaking ethnic groups in the Horn of Africa are not represented in the international genomic projects such as the 1000 genomes and the HapMap.

The predisposition of HLA DR-DQ haplotypes to diabetes among Somali children was indeed previously reported by two studies. First, Oilinki et al. ${ }^{23}$ found that DR3-DQ2 was the dominating HLA haplotype in Somali children with T1D in Finland. In another study, Sunni et al. ${ }^{22}$ studied Somali children with T1D in Minnesota 
(US) and found that most of them (93\%) carried the T1D susceptibility HLA allele DRB1*03:01. Another HLA allele, DRB3*02:02, which was frequent in our Somali study population (Table 2) forms haplotype with DRB1*03:01 and was previously found to be associated with $\mathrm{T}^{51} \mathrm{D}^{51}$. Moreover, a Swedish study ${ }^{52}$ investigating T1D risk among children compared 35,756 children of Horn of African ancestry (Somalia, Ethiopia and Eritrea) with 1,666,051 children of native Swedish parents and found that children with Horn of African ancestry had significantly increased risk for T1D. The risk was highest among Eritreans, followed by Somalis and lowest among Ethiopians. Unfortunately, we could not find enough HLA data for Eritreans in the literature to include in our analysis and it is therefore not inconceivable that T1D predisposing HLA alleles are also prevalent in Eritrea. These evidences suggest that Somali children are genetically predisposed to juvenile autoimmune diabetes, with HLA as an important contributing factor. Thus, physicians and other healthcare workers should be aware of the genetic predisposition to T1D among Somali children when performing differential diagnosis, warranting inclusion of blood sugar tests even in cases in which classical diabetes symptoms cannot be clearly observed. In addition to T1D, a study by D'Souza et al. identified an unusual form of autoimmune hepatitis observed in Somali men living in the United Kingdom ${ }^{53}$. Although the authors emphasized their study to be underpowered, they speculated that HLA types among Somalis appear to differ from those seen in other ethnic groups, an observation we corroborate in this study.

This study has some limitations. One major limitation is the absence of cases in the analyses. For investigating the role of HLA DR-DQ haplotypes in T1D risk among Somalis, a case-controlled study with sufficient power will be required. Such study should preferably also include neighboring Horn of African populations, including Djibouti and Eritrea, for whom there are no or little genomic data available. To explore mechanisms involving disease alleles or pharmacogenetic variants, approaches recently reported by Adeyemo et al. ${ }^{7}$ will be useful to employ.

In conclusion, this study provides the first publicly available Somali genomic dataset with significance to precision medicine. We report that ethnic Somalis are an East African population with unique HLA composition and that the Somali population hosts high frequencies of genetic variants with implications to T1D and pharmacogenetics. These variants should be validated in Somali patients in the future.

\section{Materials and Methods}

All methods were carried out in accordance with relevant guidelines and regulations.

Ethics statement. The study was granted with ethical permissions from the National Ethics Review Authority in Sweden (registration number: 2019-00926) as well as from the East Africa University in Bosaso, Somalia (registration number: JBA-XG-0424). Written informed consent was obtained from all study subjects.

Study population. The study recruited 95 unrelated individuals living in Bosaso city in the Northeastern region (Puntland) of Somalia. Birth places were confirmed with available documents or based on questionnaires. All participants were identified as ethnic Somalis belonging to the recognized clans of the Somali lineage. Age ranged between 18 and 65 years (57 males and 38 females). Most of the study participants (96\%) were born in Puntland. Two individuals were born in South-Central Somalia, another person was born in Northwestern Somalia (Somaliland), and one individual was born in the Somali region of Eastern Ethiopia.

Population databases. For genome-wide principal component analysis (PCA), the Somali genotype data were projected onto African - West Eurasian PC1 and PC2 data from populations in the PGG.Population database (https://pggpopulation.org/). For HLA analysis, the Allele Frequency Net Database (http://www.allelefrequencies.net/) was used to collect data on HLA frequencies for other world populations. Populations with close genetic and geographic distance were merged into a single group, e.g. Oromo, Amhara, Ethiopian Jews (=Ethiopia), Kongo Kinshasa, Central African Republic, Rwanda, Uganda (=Central Africa), Tunisia, Algeria, Morocco (=North Africa), Saudi Arabia, United Arab Emirates (=South Arabia), England, Germany, Sweden, Norway (=North Europe), Native Americans (=AMR).

DNA extraction and Genotyping. Blood was collected into PAXgene Blood DNA Tubes (Qiagen, Hilden, Germany) and DNA was extracted using QIAamp DNA Blood Midi kit (Qiagen). Subsequent to DNA quality control, samples were genotyped using the Axiom Precision Medicine Research Array (PMRA) at the Array and Analysis Facility, Uppsala University, Sweden. The PMRA enabled genotyping of nearly 900,000 genomic markers, including $>1,200$ pharmacogenetic variants, $>9,000$ HLA markers, $>2,000$ blood phenotypes, $>23,000$ variants in ClinVar, as well as other disease or immunological markers. The analysis was performed according to the protocol described in the Axiom 2.0 Assay Manual Workflow User Guide (Thermo Fisher Scientific Inc., Waltham, MA, USA). Briefly, DNA amplification was followed by fragmentation and DNA precipitation. The next steps included drying, resuspension, denaturation and hybridization of the DNA onto the arrays, which were followed by ligation, staining and scanning of the arrays. The array processing was performed using the GeneTitan MC Instrument, the GeneTitan Instrument Control software and the AGCC Portal 4.0 software (Thermo Fisher).

Data analysis. For genotyping, raw data was analyzed with the Axiom Array Analysis Suite, version 4.0.3.3 (Thermo Fisher), using the Best Practices Workflow with default settings. To infer HLA types of the Somali samples from the Axiom PMRA genotype data, the Axiom HLA Analysis 1.2 software (Thermo Fisher) was utilized with default settings. Genome-wide PCA analysis was performed using the PLINK 1.9 software (http://zzz.bwh. harvard.edu/plink $/)^{54}$. Maximum likelihood estimation of individual ancestries from the Axiom PMRA multilocus SNP genotypes was evaluated using the ADMIXTURE 1.3.0 software (http://software.genetics.ucla.edu/ admixture/ $)^{55}$. Hierarchical clustering and PCA for HLA were carried out with R software (https://www.R-project. org/). Identity by descent (IBD) analysis was performed using PLINK 1.9 and the gdsfmt as well as SNPRelate R 
packages $^{56}$. Haplotype analysis of vitamin K epoxide reductase complex subunit 1 (VKORC1) gene was performed with the Haploview 4.0 software (https://www.broadinstitute.org/haploview/haploview) ${ }^{57}$. Hardy-Weinberg equilibrium was evaluated using $C h i^{2}$ test with a significance level of $P<0.05$.

\section{Data availability}

Raw experimental files (CEL files) from the Axiom PMRA analyses are publicly available on the Array Express database (https://www.ebi.ac.uk/arrayexpress), with the accession number: E-MTAB-8331.

Received: 13 January 2020; Accepted: 17 March 2020;

Published online: 27 March 2020

\section{References}

1. Chimusa, E. R. et al. A genomic portrait of haplotype diversity and signatures of selection in indigenous southern African populations. PLoS Genet. 11, e1005052, https://doi.org/10.1371/journal.pgen.1005052 (2015).

2. Heenkenda, M. K., Lindahl, T. L. \& Osman, A. Frequency of PAR4 Ala120Thr variant associated with platelet reactivity significantly varies across sub-Saharan African populations. Blood 132, 2103-2106, https://doi.org/10.1182/blood-2018-05-852335 (2018).

3. Tishkoff, S. A. \& Williams, S. M. Genetic analysis of African populations: human evolution and complex disease. Nat. Rev. Genet. 3, 611-621, https://doi.org/10.1038/nrg865 (2002).

4. Fan, S. et al. African evolutionary history inferred from whole genome sequence data of 44 indigenous African populations. Genome Biol. 20, 82, https://doi.org/10.1186/s13059-019-1679-2 (2019).

5. Lorente-Galdos, B. et al. Whole-genome sequence analysis of a Pan African set of samples reveals archaic gene flow from an extinct basal population of modern humans into sub-Saharan populations. Genome Biol. 20, 77, https://doi.org/10.1186/s13059-019-16845 (2019).

6. Tishkoff, S. A. et al. The genetic structure and history of Africans and African Americans. Science 324, 1035-1044, https://doi. org/10.1126/science.1172257 (2009).

7. Adeyemo, A. A. et al. ZRANB3 is an African-specific type 2 diabetes locus associated with beta-cell mass and insulin response. Nat. Commun. 10, 3195, https://doi.org/10.1038/s41467-019-10967-7 (2019).

8. Onengut-Gumuscu, S. et al. Type 1 Diabetes Risk in African-Ancestry Participants and Utility of an Ancestry-Specific Genetic Risk Score. Diabetes Care 42, 406-415, https://doi.org/10.2337/dc18-1727 (2019).

9. Gurdasani, D. et al. The African Genome Variation Project shapes medical genetics in Africa. Nature 517, 327-332, https://doi. org/10.1038/nature13997 (2015).

10. Gandini, F. et al. Mapping human dispersals into the Horn of Africa from Arabian Ice Age refugia using mitogenomes. Sci. Rep. 6, 25472, https://doi.org/10.1038/srep25472 (2016).

11. Musilova, E. et al. Population history of the Red Sea-genetic exchanges between the Arabian Peninsula and East Africa signaled in the mitochondrial DNA HV1 haplogroup. Am. J. Phys. Anthropol. 145, 592-598, https://doi.org/10.1002/ajpa.21522 (2011).

12. Pagani, L. et al. Ethiopian genetic diversity reveals linguistic stratification and complex influences on the Ethiopian gene pool. Am. J. Hum. Genet. 91, 83-96, https://doi.org/10.1016/j.ajhg.2012.05.015 (2012).

13. Shriner, D., Tekola-Ayele, F., Adeyemo, A. \& Rotimi, C. N. Ancient Human Migration after Out-of-Africa. Sci. Rep. 6, 26565, https:// doi.org/10.1038/srep26565 (2016).

14. Gutherz, X., Cros, J.-P. \& Lesur, J. The discovery of new rock paintings in the Horn of Africa: The rock shelters of Las Geel, Republic of Somaliland. J. Afr. Archaeology 1, 227-236 (2003).

15. Sada, M. Mapping the Archaeology of Somaliland: Religion, Art, Script, Time, Urbanism, Trade and Empire. Afr. Archaeol. Rev. 32, $11-136(2015)$.

16. Hodgson, J. A., Mulligan, C. J., Al-Meeri, A. \& Raaum, R. L. Early back-to-Africa migration into the Horn of Africa. PLoS Genet. 10, e1004393, https://doi.org/10.1371/journal.pgen.1004393 (2014).

17. Sanchez, J. J., Hallenberg, C., Borsting, C., Hernandez, A. \& Morling, N. High frequencies of Y chromosome lineages characterized by E3b1, DYS19-11, DYS392-12 in Somali males. Eur. J. Hum. Genet. 13, 856-866, https://doi.org/10.1038/sj.ejhg.5201390 (2005).

18. Hedman, M., Palo, J. U. \& Sajantila, A. X-STR diversity patterns in the Finnish and the Somali population. Forensic Sci. Int. Genet. 3 , 173-178, https://doi.org/10.1016/j.fsigen.2009.02.005 (2009).

19. Neuvonen, A. M., Palo, J. U., Hedman, M. \& Sajantila, A. Discrimination power of Investigator DIPplex loci in Finnish and Somali populations. Forensic Sci. Int. Genet. 6, e99-102, https://doi.org/10.1016/j.fsigen.2011.09.005 (2012).

20. Tillmar, A. O., Backstrom, G. \& Montelius, K. Genetic variation of 15 autosomal STR loci in a Somali population. Forensic Sci. Int. Genet. 4, e19-20, https://doi.org/10.1016/j.fsigen.2009.01.004 (2009).

21. Tillmar, A. O. et al. Analysis of linkage and linkage disequilibrium for eight X-STR markers. Forensic Sci. Int. Genet. 3, 37-41, https:// doi.org/10.1016/j.fsigen.2008.09.006 (2008).

22. Sunni, M. et al. Predominance of DR3 in Somali children with type 1 diabetes in the twin cities, Minnesota. Pediatr. Diabetes 18, 136-142, https://doi.org/10.1111/pedi.12369 (2017).

23. Oilinki, T., Otonkoski, T., Ilonen, J., Knip, M. \& Miettinen, P. J. Prevalence and characteristics of diabetes among Somali children and adolescents living in Helsinki, Finland. Pediatr. Diabetes 13, 176-180, https://doi.org/10.1111/j.1399-5448.2011.00783.x (2012).

24. Raffan, E. et al. Founder effect in the Horn of Africa for an insulin receptor mutation that may impair receptor recycling. Diabetologia 54, 1057-1065, https://doi.org/10.1007/s00125-011-2066-z (2011).

25. Sanchez, J. J. et al. Carrier frequency of a nonsense mutation in the adenosine deaminase (ADA) gene implies a high incidence of ADA-deficient severe combined immunodeficiency (SCID) in Somalia and a single, common haplotype indicates common ancestry. Ann. Hum. Genet. 71, 336-347, https://doi.org/10.1111/j.1469-1809.2006.00338.x (2007).

26. Sistonen, P., Koistinen, J. \& Aden Abdulle, O. Distribution of blood groups in the East African Somali population. Hum. Hered. 37, 300-313, https://doi.org/10.1159/000153722 (1987).

27. Abdi, A. A. \& Osman, A. Prevalence of common hereditary risk factors for thrombophilia in Somalia and identification of a novel Gln544Arg mutation in coagulation factor V. J. Thromb. Thrombolysis 44, 536-543, https://doi.org/10.1007/s11239-017-1543-8 (2017).

28. Singh, P. P., Singh, M. \& Mastana, S. S. APOE distribution in world populations with new data from India and the UK. Ann. Hum. Biol. 33, 279-308, https://doi.org/10.1080/03014460600594513 (2006).

29. Abondio, P. et al. The Genetic Variability of APOE in Different Human Populations and Its Implications for Longevity. Genes (Basel) 10, https://doi.org/10.3390/genes10030222 (2019).

30. Zekraoui, L. et al. High frequency of the apolipoprotein $\mathrm{E} * 4$ allele in African pygmies and most of the African populations in subSaharan Africa. Hum. Biol. 69, 575-581 (1997).

31. Corbo, R. M., Scacchi, R., Rickards, O., Martinez-Labarga, C. \& De Stefano, G. F. An investigation of human apolipoproteins B and E polymorphisms in two African populations from Ethiopia and Benin. Am J Hum Biol 11, 297-304, https://doi.org/10.1002/ (SICI) 1520-6300(1999)11:3<297::AID-AJHB2>3.0.CO;2-B (1999). 
32. Troberg, J. \& Finel, M. The Polymorphic Variant P24T of UDP-Glucuronosyltransferase 1A4 and Its Unusual Consequences. Drug. Metab. Dispos. 43, 1769-1772, https://doi.org/10.1124/dmd.115.065680 (2015).

33. Benoit-Biancamano, M. O. et al. A pharmacogenetics study of the human glucuronosyltransferase UGT1A4. Pharmacogenet Genomics 19, 945-954, https://doi.org/10.1097/FPC.0b013e3283331637 (2009).

34. Loebstein, R. et al. A coding VKORC1 Asp36Tyr polymorphism predisposes to warfarin resistance. Blood 109, 2477-2480, https:// doi.org/10.1182/blood-2006-08-038984 (2007).

35. Li, T. et al. Identification of the gene for vitamin K epoxide reductase. Nature 427, 541-544, https://doi.org/10.1038/nature02254 (2004).

36. Rost, S. et al. Mutations in VKORC1 cause warfarin resistance and multiple coagulation factor deficiency type 2. Nature 427, 537-541, https://doi.org/10.1038/nature02214 (2004).

37. Osman, A., Enstrom, C., Arbring, K., Soderkvist, P. \& Lindahl, T. L. Main haplotypes and mutational analysis of vitamin K epoxide reductase (VKORC1) in a Swedish population: a retrospective analysis of case records. J. Thromb. Haemost. 4, 1723-1729, https:// doi.org/10.1111/j.1538-7836.2006.02039.x (2006).

38. Geisen, C. et al. VKORC1 haplotypes and their impact on the inter-individual and inter-ethnical variability of oral anticoagulation. Thromb. Haemost. 94, 773-779, https://doi.org/10.1160/TH05-04-0290 (2005).

39. Rieder, M. J. et al. Effect of VKORC1 haplotypes on transcriptional regulation and warfarin dose. N. Engl. J. Med. 352, 2285-2293, https://doi.org/10.1056/NEJMoa044503 (2005).

40. van Gerven, N. M. et al. HLA-DRB1*03:01 and HLA-DRB1*04:01 modify the presentation and outcome in autoimmune hepatitis type-1. Genes. Immun. 16, 247-252, https://doi.org/10.1038/gene.2014.82 (2015).

41. Aklillu, E. et al. SLCO1B1 Gene Variations Among Tanzanians, Ethiopians, and Europeans: Relevance for African and Worldwide Precision Medicine. OMICS 20, 538-545, https://doi.org/10.1089/omi.2016.0119 (2016).

42. Ahmed, J. H. et al. CYP2D6 Genotype Predicts Plasma Concentrations of Tamoxifen Metabolites in Ethiopian Breast Cancer Patients. Cancers (Basel) 11, https://doi.org/10.3390/cancers11091353 (2019).

43. Aklillu, E., Carrillo, J. A., Makonnen, E., Bertilsson, L. \& Djordjevic, N. N-Acetyltransferase-2 (NAT2) phenotype is influenced by genotype-environment interaction in Ethiopians. Eur. J. Clin. Pharmacol. 74, 903-911, https://doi.org/10.1007/s00228-018-2448-y (2018).

44. Aklillu, E., Leong, C., Loebstein, R., Halkin, H. \& Gak, E. VKORC1 Asp36Tyr warfarin resistance marker is common in Ethiopian individuals. Blood 111, 3903-3904, https://doi.org/10.1182/blood-2008-01-135863 (2008).

45. Yimer, G. et al. Pharmacogenetic \& pharmacokinetic biomarker for efavirenz based ARV and rifampicin based anti-TB drug induced liver injury in TB-HIV infected patients. PLoS One 6, e27810, https://doi.org/10.1371/journal.pone.0027810 (2011).

46. Ceballos, F. C., Hazelhurst, S. \& Ramsay, M. Runs of homozygosity in sub-Saharan African populations provide insights into complex demographic histories. Hum. Genet. 138, 1123-1142, https://doi.org/10.1007/s00439-019-02045-1 (2019).

47. Shahin, M. H. et al. VKORC1 Asp36Tyr geographic distribution and its impact on warfarin dose requirements in Egyptians. Thromb. Haemost. 109, 1045-1050, https://doi.org/10.1160/TH12-10-0789 (2013).

48. Pan, K. L. et al. Effects of Non-Vitamin K Antagonist Oral Anticoagulants Versus Warfarin in Patients With Atrial Fibrillation and Valvular Heart Disease: A Systematic Review and Meta-Analysis. J Am Heart Assoc 6, https://doi.org/10.1161/JAHA.117.005835 (2017).

49. Choo, S. Y. The HLA system: genetics, immunology, clinical testing, and clinical implications. Yonsei Med. J. 48, 11-23, https://doi. org/10.3349/ymj.2007.48.1.11 (2007).

50. Noble, J. A. et al. HLA class I and genetic susceptibility to type 1 diabetes: results from the Type 1 Diabetes Genetics Consortium. Diabetes 59, 2972-2979, https://doi.org/10.2337/db10-0699 (2010).

51. Erlich, H. A. et al. Next generation sequencing reveals the association of DRB3*02:02 with type 1 diabetes. Diabetes 62, 2618-2622, https://doi.org/10.2337/db12-1387 (2013).

52. Hjern, A., Soderstrom, U. \& Aman, J. East Africans in Sweden have a high risk for type 1 diabetes. Diabetes Care 35, 597-598, https:// doi.org/10.2337/dc11-1536 (2012).

53. D’Souza, R., Sinnott, P., Glynn, M. J., Sabin, C. A. \& Foster, G. R. An unusual form of autoimmune hepatitis in young Somalian men. Liver Int. 25, 325-330, https://doi.org/10.1111/j.1478-3231.2005.01088.x (2005).

54. Purcell, S. et al. PLINK: a tool set for whole-genome association and population-based linkage analyses. Am. J. Hum. Genet. 81, 559-575, https://doi.org/10.1086/519795 (2007).

55. Alexander, D. H., Novembre, J. \& Lange, K. Fast model-based estimation of ancestry in unrelated individuals. Genome Res. 19, 1655-1664, https://doi.org/10.1101/gr.094052.109 (2009).

56. Zheng, X. et al. A high-performance computing toolset for relatedness and principal component analysis of SNP data. Bioinformatics 28, 3326-3328, https://doi.org/10.1093/bioinformatics/bts606 (2012).

57. Barrett, J. C., Fry, B., Maller, J. \& Daly, M. J. Haploview: analysis and visualization of LD and haplotype maps. Bioinformatics 21, 263-265, https://doi.org/10.1093/bioinformatics/bth457 (2005).

\section{Acknowledgements}

We are grateful to Menikae K. Heenkenda for her assistance in some stages of this study. Open access funding provided by Linköping University.

\section{Author contributions}

A.O. and A.A.A. conceptualized and designed the project. A.A.A. recruited study participants and collected the samples. J.J. and A.O. analysed the data. A.O. supervised the study. A.O. and J.J. wrote the paper. M.A. contributed intellectually to the project.

\section{Competing interests}

The authors declare no competing interests.

\section{Additional information}

Supplementary information is available for this paper at https://doi.org/10.1038/s41598-020-62645-0.

Correspondence and requests for materials should be addressed to A.O.

Reprints and permissions information is available at www.nature.com/reprints.

Publisher's note Springer Nature remains neutral with regard to jurisdictional claims in published maps and institutional affiliations. 
(c) (i) Open Access This article is licensed under a Creative Commons Attribution 4.0 International License, which permits use, sharing, adaptation, distribution and reproduction in any medium or format, as long as you give appropriate credit to the original author(s) and the source, provide a link to the Creative Commons license, and indicate if changes were made. The images or other third party material in this article are included in the article's Creative Commons license, unless indicated otherwise in a credit line to the material. If material is not included in the article's Creative Commons license and your intended use is not permitted by statutory regulation or exceeds the permitted use, you will need to obtain permission directly from the copyright holder. To view a copy of this license, visit http://creativecommons.org/licenses/by/4.0/.

(C) The Author(s) 2020 